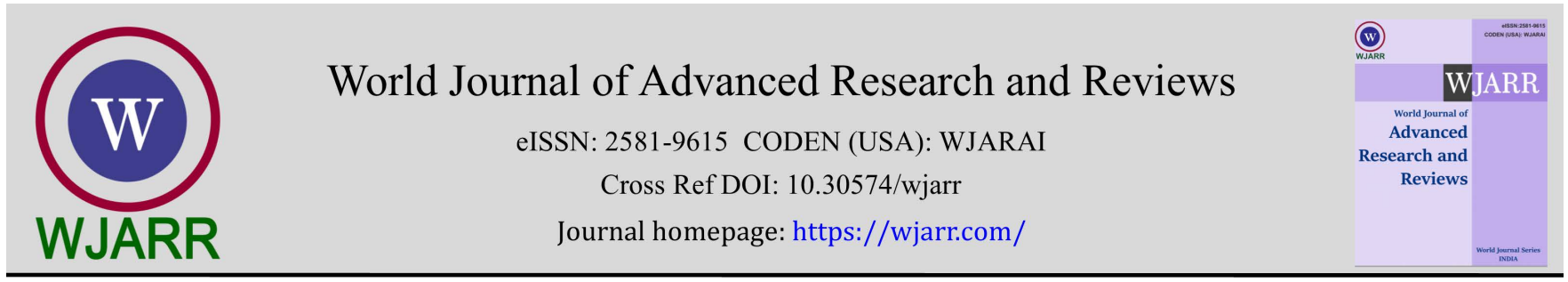

(RESEARCH ARTICLE)

Check for updates

\title{
Prediction of labor outcome by measuring the angle of progression using two dimensional trans-perineal Ultrasound: A prospective cohort study
}

\author{
Diaa Abdelhalim ${ }^{1}$, Hussein Abolmakarem ${ }^{1}$ and Mohamed Hassan 2 , \\ ${ }^{1}$ Al Galaa teaching Hospital, Cairo, Egypt. \\ 2 New Cairo General Hospital, Obstetrics \& gynecology department, Cairo, Egypt.
}

World Journal of Advanced Research and Reviews, 2021, 12(03), 200-204

Publication history: Received on 05 November 2021; revised on 10 December 2021; accepted on 12 December 2021

Article DOI: https://doi.org/10.30574/wjarr.2021.12.3.0675

\begin{abstract}
Background: Failure of descent due to fetal malposition is one of the most common indications for performing surgical deliveries. It has recently been suggested that trans-perineal intra-partum ultrasonography may be useful in assessing fetal head engagement, position and station as well as it's reliable, cheap, painless and effective tool. Measuring the 'angle of progression' could assist in the obstetrician's decision regarding mode of delivery.
\end{abstract}

Objectives: The primary outcome is to use the AOP to develop a predictive model for the probability of successful vaginal delivery. Secondary outcomes including assessment of possibly successful VBAC in previous one CS women.

Methods: We recruited 500 women in labor. For each woman, a (TPU) was performed to measure the AOP in late first and second stages of labor. We compared AOP between women who delivered fetuses through vaginal route to those who delivered by CS.

Results: Through 467 women included in the study, AOP was significantly Higher in spontaneous vaginal delivery group (with cut off $\left.123^{\circ} \pm 8.5^{\circ}\right)$ as compared with women delivered by vacuum or by CS $\left(113^{\circ} \pm 10.5^{\circ}\right)(\mathrm{P}=0.003)$. The VBAC as another secodary outcome seems to be insignificant.

Conclusions: TPU is safe, non-invasive and easily preformed technique which is useful to predict labor outcomes.

Keywords: Trans-perineal Ultrasound; Angle of progression; Vaginal delivery; Cesarean section

\section{Introduction}

In recent years the rate of Cesarean sections has increased dramatically to approximately $31 \%$ in the USA and up to $50 \%$ in other countries, and either failure of descent or fetal malposition are very common indications for performing surgical deliveries [1].

Traditionally, the assessment and management of a woman in labor is based upon clinical findings [2]. The diagnosis of arrest of labor and decisions regarding the timing or type of intervention rely mostly on digital evaluation of cervical dilatation and fetal head station and position $[3,4]$.

Ultrasound imaging has already been reported as an adjunctive method for evaluating the level of the fetal presenting part within the maternal pelvis $[5,6]$.

\footnotetext{
${ }^{*}$ Corresponding author: Mohamed Hassan

New Cairo General Hospital, Obstetrics \& gynecology department, Cairo, Egypt.

Copyright $(2021$ Author(s) retain the copyright of this article. This article is published under the terms of the Creative Commons Attribution Liscense 4.0.
} 
Several studies have demonstrated that ultrasound examination is more accurate and Reproducible than clinical examination in the diagnosis of fetal head position and station and in the prediction of arrest of labor $[7,8,9]$.

The angle of Progression AOP was described by Barbera et al. for the first time as an accurate and reproducible parameter, and its reliability is independent of the fetal head station or on the operator experience in performing ultrasound exam, and she used the term (Angle of Head Descent) [10].

Measuring the angle formed between a line placed through the long axis of the symphysis pubis and a second line drawn from the inferior edge of the symphysis tangentially to the fetal skull (the 'angle of progression') on Trans-perineal ultrasound images could assist in the obstetrician's decision to attempt a vaginal delivery or Cesarean section [11].

In the second stage of labor, the fetus in an occiput anterior position (OAP), an angle of progression of $120{ }^{\circ}$ or greater was always associated with subsequent successful spontaneous vaginal delivery, while in Pregnancies ending in Cesarean section the mean angle of descent measured at last TPU examination was only $108{ }^{\circ}$. [10].

Many studies had been conducted to assess the correlation between AOP and successful vaginal delivery, and their results were very near to Barbera et al. $[12,13]$.

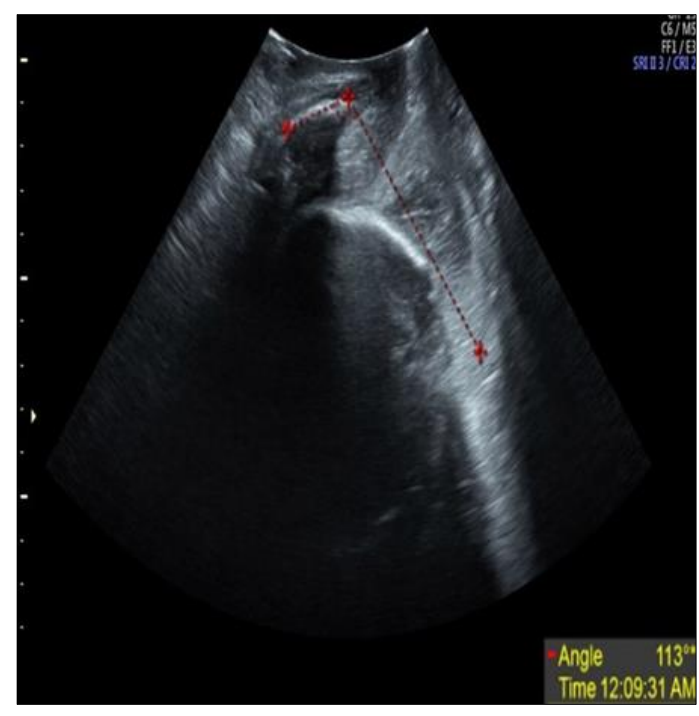

Figure 1 Denomestrated the measurement of AOP in second stage

The aim of the present study was to use the angle of progression measured by trans-perineal ultrasound to develop a more accurate predictive model for the probability of vaginal delivery and/or successful vacuum extraction at the second stage of labor. Secondary outcomes include assessment of possibly successful VBAC in previous one CS women and to evaluate the duration of second stage of labor as well.

\section{Patients \& Methods}

\subsection{Design \& population}

This was a prospective observational study conducted in a tertiary-level (Al Galaa teaching hospital) between April 2019 to April 2020.

The study included 500 pregnant women who attended the labor ward and emergency unit of the hospital and they had been selected after fulfilling these criteria:

\subsubsection{Inclusion criteria}

- $\quad$ Singleton pregnancy.

- Viable full term fetus (Gestational age 37-42 weeks).

- Vertex presentation. 
- Second stage of labor (full cervical dilatation).

\subsubsection{Exclusion criteria}

- Any emergency situation in second stage of labor due to either maternal or fetal reasons (Intra-partum bleeding or fetal distress).

- Any obvious indication for C.S. at the time of recruitment.

- Women with history of pelvic traumas or previous pelvic surgeries.

For each woman, a trans-perineal ultrasound (TPU) was performed using (Voluson P8 GE ultrasound machine) to measure angle of progression AOP in late first and second stages of labor in regular sequence. All women gave a verbal consent prior to study beginning and all of them had been examined by digital vaginal examination before ultrasound assessment. We compared AOP between women who delivered fetuses through vaginal route to those who delivered by CS.

\subsection{Statistical Analysis}

Univariate analysis will be used to compare the two groups. Continues data will be calculated with the Students T-test or Mann-Whitney U test and presented as mean and SD for normally distributed data and median (range) for those not normally distributed. Categorical data will be calculated with the Chi-square test and presented in mean and percentages.

Receiver-operating characteristics (ROC) curves were constructed in order to estimate the accuracy of ultrasound parameters in predicting vaginal delivery. The area under the ROC curve (AUC) was used as discriminator and considered to have discriminatory potential if the lower limit of the $95 \% \mathrm{CI}$ interval exceeded 0.5 .

Multi-logistic regression analysis will be used to evaluate the association between the angle of progression and possibility of a vaginal delivery or successful vacuum extraction. Vaginal delivery will be used as the outcome variable and the angle of progression as the independent variable. Statistical analysis was performed using SPSS (version 18.0 for Windows). $\mathrm{P}<0.05$ was considered statistically significant

\subsection{Ethical Considerations}

The study was approved by the Ethics Committee of Al Galaa hospital and was conducted in accordance with the Declaration of Helsinki and the International Conference on Harmonization Guidelines for Good Clinical Practice. Informed consent was obtained from all eligible women before starting the study.

\section{Results}

Overall, 467 women were included in the study (33 women's data were lost). Among these, 398 women (85.2\%) delivered via spontaneous vaginal route, while $9(1.9 \%)$ delivered by vacuum extraction, and the rest of 60 women $\left(12.8 \%\right.$ ) delivered by CS. AOP was significantly Higher in spontaneous vaginal delivery group (with cut off $123^{\circ} \pm 8.5^{\circ}$ ) as compared with women who delivered foetuses by $\mathrm{CS}\left(113^{\circ} \pm 10.5^{\circ}\right)(\mathrm{P}=0.003)$. The Area under Receiver-operating characteristics curve was $0.731 \pm 0.070$ (95\% C.I. 0.594-0.869) with an estimated best cut-off range of $121.5^{\circ}$ (sensitivity 71.8\% and specificity 66.7\%). Area under Receiver-operating characteristics curve for the prediction of Caesarean delivery was $0.866 \pm 0.054$ (95\% C.I. 0.761-0.972). The time of second stage evaluation as secondary outcome confirmed that smallest angles $\left(\leq 125^{\circ}\right.$ ) had a markedly greater time to delivery (median 45 (range, 30-60) min) than the other groups $(125 \circ-170 \circ:$ median, 27.5 (range, 15-35) min; 171॰-200॰: median, 16 (range, 10-22) min; > $200 \circ:$ median, 5 (range, 1-9) min. The VBAC as another secondary outcome seems to be insignificant.

\section{Discussion}

Despite significant progress in clinical obstetrics, the evaluation of fetal head engagement and strategies for monitoring the progress of the fetal head through the birth canal still remain subjective and inexact processes.

Ultrasound in active labor is not yet used widely, even though studies have shown that it is more precise and reproducible than clinical examination. Ultrasound allows objective measurement and precise documentation of findings obtained during the examination. 
Ultrasound is likely to be of significant use in labor in two situations either suspected delay of first or second stage (with particular recommendations to measure AOP) or Potential need for performance of OVD [14].

\subsection{Outcomes}

In our study, 467 women were recruited (33 women's data were missed). We had 398 women (85.2\%) delivered via spontaneous vaginal route, while $9(1.9 \%)$ delivered by vacuum extraction, and the rest of 60 women (12.8\%) delivered by CS. AOP was obviously narrower in CS delivery group $\left(113^{\circ} \pm 10.5^{\circ}\right)$ as compared with women who delivered fetuses vaginally $\mathrm{CS}\left(123^{\circ} \pm 8.5^{\circ}\right)(\mathrm{P}=0.003)$. the statistics estimated best cut-off degree of AOP for successful safe vaginal delivery to be $121.5^{\circ}$.

The time of second stage evaluation as secondary outcome confirmed that small angles $\left(\leq 125^{\circ}\right)$ had a markedly greater time to delivery.

The VBAC as another secondary outcome seems to be insignificantly affected the process of successful vaginal delivery if the AOP was adequate.

\subsection{Strengths \& Limitations}

The study sample size is relatively large to evaluate properly the AOP and its relation to the mode of delivery which will provide a different experience to obstetricians in their clinical practice of vaginal delivery.

The study also recruited women for vaginal delivery with history of previous one cesarean section due to nonpermanent reason and gave a reliable idea about high successful vaginal delivery chances with wide AOP.

On the other hand, important point to consider is that the application of intra-partum ultrasound in daily clinical practice in labor ward is still limited.

This maybe because of lacking experience and familiarity with TPU techniques from medical staff.

\section{Conclusion}

Based on our results, we concluded that measurement of AOP in active labor is a simple, easy, non-invasive reliable method to predict the possible mode of delivery, with a cutoff degree of 121.5 for safe uncomplicated vaginal delivery.

We recommend further studies targeting other valuable fetal-pelvic diameters like Head Perineal Distance (HPD), Head Symphysis Diameter (HSD), Midline Angle (MLA) and Antero-posterior Pelvic Diameter (APP) which will help us to understand the mechanisms of labor for different fetal presentations and positions as well as predict the mode of delivery.

Additionally, using of the 3D ultrasound instead of regular 2D may add valuable information which may help in better management of vaginal deliveries through accurate measurements of different pelvic diameters particularly the anteroposterior pelvic area.

\section{Compliance with ethical standards}

\section{Acknowledgments}

I wish to show my appreciation to my colleagues and to thank all people in Al-Galaa Teaching Hospital \& New Cairo Hospital for their unlimited support and help.

\section{Disclosure of conflict of interest}

There is no conflict of interest.

\section{Statement of informed consent}

Informed consents were obtained from all individual participants involved in the study. 


\section{References}

[1] Kolas T, Hofoss D, Daltveit AK, Nilsen ST, Henriksen T, ${ }^{\circ}$ Hager R,Ingemarsson I, Øian P. Indications for cesarean "deliveries in Norway. Am J Obstet Gynecol. 2003; 188: 864-870.

[2] Hamilton EF, Simoneau G, Ciampi A, Warrick P, Collins K, Smith S, Garite TJ. Descent of the fetal head (station) during the first stage of labor. Am J Obstet Gynecol. 2016; 214: 360.e1-6.

[3] Leveno KJ, Nelson DB, McIntire DD. Second-stage labor: how long is too long? Am J Obstet Gynecol. 2016; 214: 484-489.

[4] Oboro VO, Tabowei TO, Bosah JO. Fetal station at the time of labor arrest and risk of caesarean delivery. J Obstet Gynaecol. 2005; 25: 20-22.

[5] Sherer DM, Abulafia 0. Intrapartum assessment of fetal head engagement: comparison between transvaginal digital and transabdominal ultrasound determinations. Ultrasound Obstet Gynecol. 2003; 21: 430-436.

[6] Heinrich W, Dudenhausen J, Fuchs I, Kamena A, Tutschek B. Intrapartum translabial ultrasound (ITU): sonographic landmark and correlation with successful vacuum extraction. Ultrasound Obstet Gynecol. 2006; 28: 753-760.

[7] Akmal S, Kametas N, Tsoi E, Hargreaves C, Nicolaides KH. Comparison of transvaginal digital examination with intrapartum sonography to determine fetal head position before instrumental delivery. Ultrasound Obstet Gynecol. 2003; 21: 437-440.

[8] Tutschek B, Torkildsen EA, Eggebo TM. Comparison between ultrasound parameters and clinical examination to assess fetal head station in labor. Ultrasound Obstet Gynecol. 2013; 41: 425-429.

[9] Dietz HP, Lanzarone V. Measuring engagement of the fetal head: validity and reproducibility of a new ultrasound technique. Ultrasound Obstet Gynecol. 2005; 25: 165-168.

[10] Barbera AF, Pombar X, Perugino G, Lezotte DC, Hobbins JC. A new method to assess fetal head descent in labor withtransperineal ultrasound. Ultrasound Obstet Gynecol. 2009; 33: 313-319.

[11] Kalache KD, Duckelmann AM, Michaelis SA, Lange J, Cichon G, Dudenhausen JW. Transperineal ultrasound imaging inprolonged second stage of labor with occipito anterior presenting fetuses: how well does the 'angle of progression' predict the mode of delivery. Ultrasound Obstet Gynecol. 2009; 33: 326-330.

[12] Amin M, Eltomey M, El-Dorf A. Role of transperineal ultrasound measurements in women with prolonged second stage of labour as predictors of the mode of delivery. 2014; EJRNM 45: 1295-1299.

[13] Kamel R, Negm S, Montaguti E, Dodaro M, Brunelli E, Di Donna G, Soliman E, Sharaf MF, ElHarty A, Youssef A. Reliability of transperineal ultrasound for the assessment of the angle of progression in labor using parasagittal approach versus midsagittal approach, The Journal of Maternal-Fetal \& Neonatal Medicine. 2019.

[14] Copyright (C) 2018 ISUOG. Published by John Wiley \& Sons Ltd. Ultrasound Obstet Gynecol. 2018; 52: 128-139. 